\title{
NORMAL VECTOR FIELDS ON MANIFOLDS ${ }^{1}$
}

\author{
W. S. MASSEY
}

1. Introduction. Let $M^{n}$ be a compact, connected, orientable, differentiable, $n$-dimensional manifold which is imbedded differentiably (without self intersections) in Euclidean $m$-space, $R^{m}$. This paper is concerned with the following question: Does there exist a continuous field of nonzero normal vectors to $M^{n}$ ?

2. Statement of results. We will be concerned with cohomology groups having as coefficients the integers, $Z$, or the integers mod 2, $Z_{2}$. If $M^{n}$ is an $n$-dimensional differentiable manifold, then $w_{i} \in H^{i}\left(M^{n}, Z_{2}\right)$ denotes the $i$ th Stiefel-Whitney class of the tangent bundle, and $\bar{w}_{i} \in H^{i}\left(M^{n}, Z_{2}\right)$ denotes the $i$ th dual Stiefel-Whitney class. Our first theorem gives a necessary condition for the existence of a normal vector field.

Theorem I. Suppose the compact, orientable n-manifold $M^{n}$ is imbedded differentiably in $(n+k+1)$-dimensional Euclidean space, $R^{n+k+1}$, where $k \geqq n / 2$. If there exists a continuous field of nonzero normal vectors, then

$$
\bar{w}_{k} \cdot \bar{w}_{n-k}=b \cdot \bar{w}_{n-k}+\mathrm{Sq}^{n-k} b
$$

for some $b \in H^{k}\left(M^{n}, Z\right)$.

Addendum to Theorem I. Under the above hypotheses,

$$
b \cdot \bar{w}_{n-k}+\mathrm{Sq}^{n-k} b=0
$$

for any $b \in H^{k}\left(M^{n}, Z\right)$ in case $k=n-2, n-3, n-4$, or $n-5$. Hence in this case the condition of the theorem reduces to

$$
\bar{w}_{k} \cdot \bar{w}_{n-k}=0 .
$$

ExAmples. Let $P_{n}(Q)$ denote $n$-dimensional quaternionic projective space ( $4 n$ real dimensions) and $\Pi$ the Cayley projective plane. By recent results of I. M. James [3], the following differentiable imbeddings are possible:

$$
\begin{array}{r}
P_{2}(Q) \subset R^{13}, \\
P_{4}(Q) \subset R^{29}, \\
\Pi \subset R^{25} .
\end{array}
$$

Received by the editors April 4, 1960.

1 During the preparation of this paper, the author was partially supported by a grant from the National Science Foundation. 
The Stiefel-Whitney classes of these manifolds can easily be computed by the method of $\mathrm{Wu}[10]$. In each case, the above theorem may be applied to show that there does not exist a continuous field of nonzero normal vectors.

Our next theorem asserts that for $k=n-2, n>4$, the condition of Theorem I is also sufficient.

Theorem II. Assume the compact, orientable n-manifold $M^{n}$ is differentiably imbedded in $R^{2 n-1}$ and $n>4$. Then $M^{n}$ admits a continuous field of nonzero normal vectors if and only if $\bar{w}_{2} \cdot \bar{w}_{n-2}=0$.

REMARKs. (1) In case $n=4$, it is easy to give a necessary and sufficient condition for the existence of a normal vector field using an earlier theorem of the author [5, Theorem V].

(2) It may be true that any orientable $n$-manifold can be differentiably imbedded in $R^{2 n-1}$; at present, no counter-examples are known.

(3) This condition for the existence of a normal vector field depends only on the homotopy type of $M^{n}$, not on the differentiable structure on $M^{n}$ or the particular imbedding in $R^{2 n-1}$.

(4) Since $\bar{w}_{2} \cdot \bar{w}_{n-2}$ is essentially a Stiefel-Whitney number of $M^{n}$, the existence of a normal vector field is a property of the cobordism class of $M^{n}$; i.e., if $M_{1}^{n}$ and $M_{2}^{n}$ are cobordant, and both can be imbedded in $R^{2 n-1}$, then both or neither admit a normal vector field. From this it follows that for $n=6$ or 7 , there always exists a normal vector field for $M^{n} \subset R^{2 n-1}$ (the Thom cobordism groups $\Omega_{6}$ and $\Omega_{7}$ are both 0 ).

(5) By Corollary 2 to Theorem I of [8], if $\bar{w}_{n-2} \neq 0$ for an orientable $n$-manifold, then $n=2^{k}\left(2^{h}+1\right)$ for non-negative integers $h$ and $k$, with the cases $k=1, h>0$ and $h=1, k \geqq 0$ ruled out. Thus $\bar{w}_{n-2} \cdot \bar{w}_{2}=0$ except possibly for these values of $n$.

Examples. (1) By a result of Hopf and I. M. James [3], $n$-dimensional real projective space, $P_{n}(R)$, can be imbedded differentiably in $R^{2 n-1}$ for $n$ odd. Using the above theorem, it is readily seen that there is always a continuous field of nonzero normal vectors for such an imbedding.

(2) By another result of I. M. James, $n$-dimensional complex projective space, $P_{n}(C)$ (a $2 n$-dimensional manifold), can be imbedded differentiably in $R^{4 n-1}$. Using Theorem II, one readily shows that there exists a normal vector field to $P_{n}(C)$ imbedded in $R^{4 n-1}$, if and only if $n$ is not a power of 2 .

In both of these examples, one can compute the Stiefel-Whitney classes by the method of $\mathrm{Wu}[10]$.

The next theorem is concerned with imbeddings $M^{n} \subset R^{2 n-2}$. Recall that the first obstruction to a cross section of the bundle of unit 
normal vectors (i.e., the Euler-Poincaré class of the normal bundle) always vanishes for an orientable $M^{n}$; this is a theorem of Seifert and Whitney.

Theorem III. For a compact, orientable $M^{n}, n>5$, differentiably imbedded in $R^{2 n-2}$, the second obstruction to the existence of a continuous field of unit normal vectors is a unique element of $H^{n-1}\left(M^{n}, Z_{2}\right)$.

Unfortunately, we have not been able to identify this second obstruction with any characteristic class. It is quite conceivable that it depends on the imbedding; perhaps it is always 0 .

THEOREM IV. For any differentiable imbedding of a sphere in Euclidean space (of any dimension) there exists a continuous field of nonzero normal vectors.

This result was pointed out to the author by M. Kervaire; it is an easy consequence of a previous paper [7].

3. Application to the immersion of manifolds in Euclidean space. By a theorem of M. Hirsch, [2], if a manifold $M^{n}$ can be immersed in $R^{n+k}$ with a field of normal vectors, then it can be immersed in $R^{n+k-1}$. If we combine this result with Theorem II above, the following is obtained:

Theorem V. Let $M^{n}$ be a compact orientable $n$-manifold $(n>4)$ which can be imbedded differentiably in $R^{2 n-1}$. If $\bar{w}_{2} \cdot \bar{w}_{n-2}=0$, then $M^{n}$ can be immersed in $R^{2 n-2}$.

EXAmples. For $n$ odd and $n>1, n$-dimensional real projective space $P_{n}(R)$ can be immersed in $R^{2 n-2}$; for $n$ even and $n$ not a power of $2, n$-dimensional complex projective space can be immersed in $R^{4 n-2}$ (compare these statements with the examples following the statement of Theorem II).

4. Proof of the theorems. First, we recall some facts about sphere bundles with vanishing characteristic class (see $[5 ; 6]$ for more details).

Let $p: E \rightarrow B$ be a fibre bundle with fibre a $k$-sphere, $S^{k}$, and $S O(k+1)$ as structure group (i.e., the bundle is orientable). Assume moreover that the characteristic class $W_{k+1} \in H^{k+1}(B, Z)$ vanishes. It then follows that the Gysin sequence breaks up into pieces of length three as follows:

$$
0 \rightarrow H^{q}(B) \stackrel{p^{*}}{\rightarrow} H^{q}(E) \stackrel{\psi}{\rightarrow} H^{q-k}(B) \rightarrow 0 .
$$

${ }^{2}$ Added in proof. Kervaire has recently indicated a slightly different proof of this result in Bull. Soc. Math. France vol. 87 (1959) p. 400. 
This holds true no matter what the coefficient group. We will be interested in the case where the coefficient group is $Z$ or $Z_{2}$.

It is also known that the group extensions involved in these exact sequences are all trivial. This can be made more precise as follows: Choose an element $a \in H^{k}(E, Z)$ such that $\psi(a)=1 \in H^{0}(B, Z)$; then for any element $u \in H^{q}(E, Z)$, there exist unique elements $u_{1}$ $\in H^{q}(B, Z)$ and $u_{2} \in H^{q-k}(B, Z)$ such that

$$
u=p^{*}\left(u_{1}\right)+a \cdot p^{*}\left(u_{2}\right) \text {. }
$$

Similarly, if $u \in H^{q}\left(E, Z_{2}\right)$, there exist unique elements $u_{1} \in H^{q}\left(B, Z_{2}\right)$ and $u_{2} \in H^{q-k}\left(B, Z_{2}\right)$ such that this same formula holds true (as usual, the cup product of an integral cohomology class and a mod 2 cohomology class is a mod 2 class). In particular, we have for $u=\mathrm{Sq}^{i}(a)$ $\in H^{k+i}\left(E, Z_{2}\right)$,

$$
\mathrm{Sq}^{i}(a)=p^{*}\left(\alpha_{i}\right)+a \cdot p^{*}\left(\beta_{i}\right)
$$

where $\alpha_{i} \in H^{k+i}\left(B, Z_{2}\right)$, and $\beta_{i} \in H^{i}\left(B, Z_{2}\right)$ are uniquely determined. Of course, $a$ is not unique; we may replace it by $a^{\prime}=a+p^{*}(b)$ for any element $b \in H^{k}(B, Z)$. Then

$$
\mathrm{Sq}^{i}\left(a^{\prime}\right)=p^{*}\left(\alpha_{i}^{\prime}\right)+a^{\prime} \cdot p^{*}\left(\beta_{i}^{\prime}\right)
$$

where

$$
\begin{aligned}
& \beta_{i}^{\prime}=\beta_{i}, \\
& \alpha_{i}^{\prime}=\alpha_{i}+\operatorname{Sq}^{i}(b)+\beta_{i} \cdot b .
\end{aligned}
$$

Thus $\beta_{i}$ is an invariant of the given sphere bundle, while only the coset of $\alpha_{i}$ modulo a certain subgroup of $H^{k+i}\left(B, Z_{2}\right)$ is an invariant. It may be shown that $\beta_{i}=w_{i}$, the $i$ th Stiefel-Whitney class $\bmod 2$; for the proof, see Liao, [4], or Massey, $[5, \S 9]$.

Suppose that the sphere buncle $p: E \rightarrow B$ admits a cross section $s: B \rightarrow E$. Then it is clear that we can choose the element $a \in H^{k}(E, Z)$ so as to satisfy the condition $s^{*}(a)=0$ in addition to the condition $\psi(a)=1$ (as a matter of fact, these conditions determine $a$ uniquely). With this choice of $a$, we have

$$
\begin{aligned}
0 & =\mathrm{Sq}^{i}\left(s^{*} a\right)=s^{*}\left(\mathrm{Sq}^{i} a\right) \\
& =s^{*}\left[p^{*}\left(\alpha_{i}\right)+a \cdot p^{*}\left(\beta_{i}\right)\right]=\alpha_{i},
\end{aligned}
$$

i.e., $\alpha_{i}=0$ for $i=1,2, \cdots, k$. Hence if $a^{\prime}=a+p^{*}(b)$, it follows from equation (4.3) that

$$
\alpha_{i}^{\prime}=\mathrm{Sq}^{i}(b)+w_{i} \cdot b
$$


We may re-state this result as follows:

Lemma 1. A necessary condition for the existence of a cross section of the $k$-sphere bundle $p: E \rightarrow B$ (with vanishing characteristic class) is that there exists an element $b \in H^{k}(B, Z)$ such that

$$
\alpha_{i}=\mathrm{Sq}^{i}(b)+w_{i} \cdot b, \quad 1 \leqq i \leqq k,
$$

where the $\alpha_{i}$ are chosen to satisfy equation (4.2).

It should be noted that it follows from the work of Liao [4] that the set of elements $\alpha_{2}^{\prime} \in H^{k+2}\left(B, Z_{2}\right)$ satisfying (4.3) for some $b \in H^{k}(B, Z)$ is precisely the second obstruction to a cross section in case $k \geqq 3$. Thus if the dimension of $B$ is $\leqq k+2$, the condition stated in Lemma 1 is also sufficient.

Let $M^{n}$ be a compact, connected, orientable differentiable manifold imbedded differentiably in $R^{n+k+1}$, and let $p: E \rightarrow M^{n}$ denote the bundle of unit normal vectors. By the argument outlined in [6], we may prove the existence of sub-algebras $A^{*}(E, Z) \subset H^{*}(E, Z)$ and $A^{*}\left(E, Z_{2}\right) \subset H^{*}\left(E, Z_{2}\right)$ which satisfy the following conditions:

(4.4) $A^{0}(E, G)=H^{0}(E, G)$,

(4.5) $H^{q}(E, G)=A^{q}(E, G)+p^{*} H^{q}(B, G)(0<q<n+k$, direct sum), (4.6) $A^{q}(E, G)=0$ for $q \geqq n+k$,

where $G=Z$ or $Z_{2}$. Moreover, the Steenrod square of an element of $A^{*}(E, Z)$ must be in $A^{*}\left(E, Z_{2}\right)$. Note also that the homomorphism $\psi$ of the Gysin sequence maps $A^{q}(E, G)$ isomorphically onto $H^{q-k}(B, G)$.

Proof of Theorem I. Assume that $M^{n}$ (compact, connected, and orientable) is differentiably imbedded in $R^{n+k+1}$, and let $p: E \rightarrow M^{n}$ denote the bundle of unit normal vectors; the fibre is a $k$-sphere. By the above remark, there exists a unique element $a \in A^{k}(E, Z)$ such that $\psi(a)=1 \in H^{0}(B, Z)$. By (4.2),

$$
\mathrm{Sq}^{i}(a)=p^{*}\left(\alpha_{i}\right)+a \cdot p\left(\bar{w}_{i}\right)
$$

for $1 \leqq i \leqq k$. Now $a \cdot \mathrm{Sq}^{n-k} a \in A^{n+k}\left(E, Z_{2}\right)$, and by (4.6), $a \cdot \mathrm{Sq}^{n-k}(a)=0$. A computation shows that

$$
\begin{aligned}
0 & =a \cdot \mathrm{Sq}^{n-k}(a)=a \cdot p^{*}\left(\alpha_{n-k}\right)+a^{2} \cdot p^{*}\left(\bar{w}_{n-k}\right) \\
& =a \cdot p^{*}\left(\alpha_{n-k}\right)+\left[p^{*}\left(\alpha_{k}\right)+a \cdot p^{*}\left(\bar{w}_{k}\right)\right] p^{*}\left(\bar{w}_{n-k}\right) \\
& =p^{*}\left(\alpha_{k} \cdot \bar{w}_{n-k}\right)+a \cdot p^{*}\left(\alpha_{n-k}+\bar{w}_{k} \cdot \bar{w}_{n-k}\right)
\end{aligned}
$$

from which it follows that 


$$
\alpha_{n-k}+\bar{w}_{k} \cdot \bar{w}_{n-k}=0
$$

or

$$
\alpha_{n-k}=\bar{w}_{k} \cdot \bar{w}_{n-k} .
$$

If we now apply Lemma 1, we obtain Theorem I.

Proof of Addendum to Theorem I. The proof is made by repeated use of Lemma 6 of [8], together with the facts that $\operatorname{Sq}^{1}(b)=0$ since $b$ is an integral cohomology class and $\bar{w}_{1}=0$ since $M^{n}$ is orientable. If $b \in H^{n-2}\left(M^{n}, Z\right)$, then

$$
b \cdot r \bar{w}_{2}=\left(\mathrm{Sq}^{1} b\right) \cdot \bar{w}_{1}+\mathrm{Sq}^{2} b=\mathrm{Sq}^{2} b,
$$

i.e., $b \cdot \bar{w}_{2}+\mathrm{Sq}^{2} b=0$. If $b \in H^{n-3}\left(M^{n}, Z\right)$, then

$$
\begin{aligned}
b \cdot \bar{w}_{3} & =\left(\mathrm{Sq}^{1} b\right) \cdot \bar{w}_{2}+\left(\mathrm{Sq}^{2} b\right) \cdot \bar{w}_{1}+\mathrm{Sq}^{3} b \\
& =\mathrm{Sq}^{3} b,
\end{aligned}
$$

i.e., $b \cdot \bar{w}_{3}+\mathrm{Sq}^{3} b=0$. In case $b \in H^{n-4}\left(M^{n}, Z\right)$,

$$
\begin{aligned}
b \cdot \bar{w}_{4} & =\left(\mathrm{Sq}^{1} b\right) \cdot \bar{w}_{3}+\left(\mathrm{Sq}^{2} b\right) \cdot \bar{w}_{2}+\left(\mathrm{Sq}^{3} b\right) \cdot \bar{w}_{1}+\mathrm{Sq}^{4} b \\
& =\left(\mathrm{Sq}^{2} b\right) \cdot \bar{w}_{2}+\mathrm{Sq}^{4} b \\
& =\left(\mathrm{Sq}^{2} \mathrm{Sq}^{2} b\right)+\mathrm{Sq}^{4} b \\
& =\mathrm{Sq}^{3} \mathrm{Sq}^{1} b+\mathrm{Sq}^{4} b=\mathrm{Sq}^{4} b,
\end{aligned}
$$

as desired, while for $b \in H^{n-5}\left(M^{n}, Z\right)$,

$$
\begin{aligned}
b \cdot \bar{w}_{5} & =\sum_{i=1}^{5}\left(\mathrm{Sq}^{i} b\right) \bar{w}_{5-i} \\
& =\left(\mathrm{Sq}^{2} b\right) \cdot \bar{w}_{3}+\left(\mathrm{Sq}^{3} b\right) \cdot \bar{w}_{2}+\mathrm{Sq}^{5} b .
\end{aligned}
$$

Now

$$
\begin{aligned}
\left(\mathrm{Sq}^{2} b\right) \cdot \bar{w}_{3} & =\left(\mathrm{Sq}^{1} \mathrm{Sq}^{2} b\right) \cdot \bar{w}_{2}+\left(\mathrm{Sq}^{2} \mathrm{Sq}^{2} b\right) \cdot \bar{w}_{1}+\mathrm{Sq}^{3} \mathrm{Sq}^{2} b \\
& =\left(\mathrm{Sq}^{3} b\right) \cdot \bar{w}_{2}
\end{aligned}
$$

since $\mathrm{Sq}^{1} \mathrm{Sq}^{2}=\mathrm{Sq}^{3}, \mathrm{Sq}^{2} \mathrm{Sq}^{2} b=\mathrm{Sq}^{3} \mathrm{Sq}^{1} b=0$, and $\mathrm{Sq}^{3} \mathrm{Sq}^{2}=0$ (by Adem's relations). Therefore

$$
b \cdot \bar{w}_{5}=\mathrm{Sq}^{5} b
$$

as desired. It is interesting to note that a similar proof shows that

$$
b \cdot \bar{w}_{6}+\mathrm{Sq}^{6} b=\mathrm{Sq}^{4} \mathrm{Sq}^{2} b
$$

for any $b \in H^{n-6}\left(M^{n}, Z\right)$, and this expression need not vanish. 
Proof of Theorem II. This is an immediate corollary of Theorem I and the result of Liao mentioned in the paragraph following Lemma 1.

Proof of Theorem III. By the result of Liao just referred to, the second obstruction to a cross section of the normal bundle for $M^{n}$ imbedded in $R^{2 n-2}$ is the set of all elements $\alpha_{2}^{\prime} \in H^{n-1}\left(M^{n}, Z_{2}\right)$ such that

$$
\alpha_{2}^{\prime}=\alpha_{2}+\mathrm{Sq}^{2} b+\bar{w}_{2} \cdot b
$$

for some $b \in H^{n-3}\left(M^{n}, Z\right)$. To prove Theorem III, one must therefore prove that

$$
\mathrm{Sq}^{2} b+\bar{w}_{2} \cdot b=0
$$

for any $b \in H^{n-3}\left(M^{n}, Z\right)$. By the Poincaré Duality Theorem with mod 2 coefficients, it suffices to prove that

$$
x \cdot\left(\mathrm{Sq}^{2} b+\bar{w}_{2} \cdot b\right)=0
$$

for any $x \in H^{1}\left(M^{n}, Z_{2}\right)$ and $b \in H^{n-3}\left(M^{n}, Z\right)$. Now

$$
\begin{aligned}
x \cdot \bar{w}_{2} \cdot b & =\bar{w}_{2} \cdot(x \cdot b)=\mathrm{Sq}^{2}(x \cdot b) \\
& =x \cdot \mathrm{Sq}^{2} b+\left(\mathrm{Sq}^{1} x\right) \cdot\left(\mathrm{Sq}^{1} b\right) \\
& =x \cdot \mathrm{Sq}^{2} b
\end{aligned}
$$

since $S q^{1} b=0$. From this it follows that $x \cdot\left(\mathrm{Sq}^{2} b+\bar{w} \cdot b\right)=0$.

Proof of Theorem IV. Assume that the $n$-sphere, $S^{n}$, is differentiably imbedded in $(n+k+1)$-dimensional Euclidean space, and let $p: E \rightarrow S^{n}$ denote the bundle of unit normal vectors (the fibre is $\left.S^{k}\right)$. Let $f: S^{n-1} \rightarrow S O(k+1)$ denote the characteristic map of this fibre bundle (as defined by Steenrod, $[9, \S 18])$, and let $\gamma \in \pi_{n-1}(S O(k+1))$ denote the homotopy class of $f$. Now consider the following commutative diagram, which appears on p. 130 of [1]:

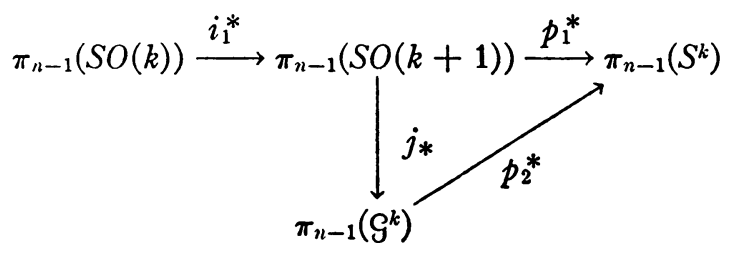

Here the top line is part of the exact sequence of the fibration $S O(k+1) / S O(k)=S^{k}, g^{k}$ denotes the space of all maps $S^{k} \rightarrow S^{k}$ of degree +1 , and $j$ denotes an inclusion map. By [7], the bundle $E$ is fibre homotopy equivalent to a product bundle; it then follows from a theorem of Dold [1] that $j_{*}(\gamma)=0$. Since $p_{1}^{*}(\gamma)=p_{2}^{*} j_{*}(\gamma)=0, \gamma$ be- 
longs to the image of $i_{1}{ }^{*}$ by exactness. But this means that the structural group of the bundle $E$ can be reduced from $S O(k+1)$ to $S O(k)$, which implies the existence of a cross section, as was to be proved.

\section{BIBLIOGRAPHY}

1. A. Dold, Über fasernweise Homotopieäquivalenz von Faserräumen, Math. Z. vol. 62 (1955) pp. 111-136.

2. M. W. Hirsch, Immersions of manifolds, Trans. Amer. Math. Soc. vol. 93 (1959) pp. $242-276$.

3. I. M. James, Some embeddings of projective spaces, Proc. Cambridge Philos. Soc. vol. 55 (1959) pp. 294-298.

4. S. D. Liao, On the theory of obstructions of fiber bundles, Ann. of Math. vol. 60 (1954) pp. 146-191.

5. W. S. Massey, On the cohomology ring of a sphere bundle, J. Math. Mech. vol. 7 (1958) pp. 265-290.

6. - On the imbeddability of the real projective spaces in Euclidean space, Pacific J. Math. vol. 9 (1959) pp. 783-789.

7. - On the normal bundle of a sphere imbedded in Euclidean space, Proc. Amer. Math. Soc. vol. 10 (1959) pp. 959-964.

8. - On the Stiefel-Whitney classes of a manifold, Amer. J. Math. vol. 82 (1960) pp. 92-102.

9. N. E. Steenrod, The topology of fibre bundles, Princeton, Princeton University Press, 1951.

10. W. T. Wu, Classes charactéristiques et i-carrés d'une variêté, C. R. Acad. Sci. Paris vol. 230 (1950) pp. 508-511.

\section{Brown UNIVERSITY}

